\title{
Effect of Inhomogeneous magnetic field on Plasma generation in a low magnetic field helicon discharge
}

\author{
Sonu Yadav, ${ }^{1, \text { a) }}$ Prabal K Chattopadhyay, (1) Kshitish K. Barada, ${ }^{2}$ Soumen Ghosh, ${ }^{3}$ \\ and Joydeep Ghosh ${ }^{1}$ \\ 1) Institute for Plasma Research, HBNI, Bhat, Gandhinagar 382428, \\ India \\ ${ }^{2)}$ Department of Physics and Astronomy, University of California, Los Angeles, \\ CA 90095, USA \\ 3) Department of Physics, University of California, San Diego, CA 92093, \\ $U S A$
}

(Dated: 11 January 2019)

The ionization efficiency of helicon plasma discharge is explored by changing the low axial magnetic field gradients near the helicon antenna. The highest plasma density is found for a most possible diverging field near the antenna by keeping the other operating condition constant. Measurement of axial wave number together with estimated radial wavenumber suggests the oblique mode propagation of helicon wave along the resonance cone boundary. Propagation of helicon wave near the resonance cone angle boundary can excite electrostatic fluctuations which subsequently can deposit energy in the plasma. This process has been shown to be responsible for peaking in density in low field helicon discharges, where the helicon wave propagates at an angle with respect to the applied uniform magnetic field. The increased efficiency can be explained on the basis of multiple resonances for multimode excitation by the helicon antenna due to the availability of a broad range of magnetic field values in the near field of the antenna when a diverging magnetic field is applied in the source.

\section{INTRODUCTION:}

Efficient plasma sources are studied for their application as an economic plasma source for micro-electronics industry ${ }^{1}$, negative ion production ${ }^{2}$, neutron generation ${ }^{3}$ and space $^{2}$ propulsion ${ }^{4}$. The helicon plasma source is one of the most efficient plasma source. This plasma source is well known for their generation of high density plasma at relatively low powers and magnetic field. Beyond the certain values of rf power and magnetic field, it is found that the helicon wave can be absorbed in the plasma and generate the high density. A large number of experiments have been conduct in various tube length and antenna geometry. Normally helicon sources operate at a magnetic field which corresponds to an electron cyclotron frequency 20 to 40 times of source frequency. This means for normal operation of the helicon source using $13.56 \mathrm{MHz}$ source $200 \mathrm{G}$ magnetic field or more is required. However, helicon sources have shown resonance abortion even around a particular low magnetic field. Here, low magnetic field $(20-30 \mathrm{G})$ means $f_{c e} \sim 5$ times source frequency.

Recently, low magnetic field helicon sources are proposed for space propulsion ${ }^{5-7}$ and also can be used as sources for semiconductor applications ${ }^{-}$as they can drastically reduce the cost of the magnet power supply and subsequently the power consumption. Resonance absorption at low magnetic fields ${ }^{9}-17$ less than $100 \mathrm{G}$ in a helicon discharge when a magnetic field is increased contrary to its behavior of monotonic increase at higher magnetic fields ${ }^{18}$. All these experiments are carried out using uniform magnetic fields near the antenna. However, Lafleur et $\mathrm{al}^{17}$ using a diverging magnetic field using one coil only near the antenna

\footnotetext{
a) Electronic mail: syadav@ipr.res.in

b) Electronic mail: pkchatto@ipr.res.in
} 
have reported a ten-fold increase in density compared to the density at zero magnetic field. While using two coils they observed that the density does not increase as much compared to while using only the single coil. In that reported experiment, the source coil current is varied and the current in the exhaust coil is kept constant at one of the values between 0-5 A (in $1 \mathrm{~A}$ steps). With the exhaust coil kept on, the non-uniformity of the magnetic field near the antenna decreases. No explanations are given for higher density production for the non-uniform magnetic field Case. Our earlier results $\frac{19}{9}$ on the density peaking phenomena in a uniform low magnetic field near antenna have shown that density increase is due to the resonance behavior while wave propagating near resonance cone. Signature of excitation of low frequency electrostatic fluctuations supports this hypothesis. As the helicon modes (radial eigenmodes) in a bounded system are discrete, only a single mode can undergo this phenomenon at a critical magnetic field when a uniform magnetic field is applied near the antenna. For a non-uniform magnetic field, there could be the resonance of different radial eigenmodes. This might lead to higher production efficiency. Hence, study the density peaking behavior in the nonuniform magnetic fields is useful.

In the present work, plasma production efficiency is studied by applying different diverging magnetic field configuration near the source (antenna). The plasma density increases with increasing the magnetic field non-uniformity near the antenna. It is observed that the plasma density is highest at particular non-uniform magnetic field configuration at low B in our experiments. These results may be thought to be due multimode cyclotron resonance due to the nonuniform magnetic fields near the antenna.

This paper is organized as follows. The experimental setup and diagnostics are described in section II followed by experimental results and discussion in section III and summary is presented in section IV.

\section{EXPERIMENTAL SETUP AND DIAGNOSTICS:}

The experiments are performed in the linear helicon plasma experimental device ( Fig. 1), which has been described before ${ }^{20,21}$. The vacuum system consists of a source and expansion chamber. The source chamber is made of borosilicate glass tube and has 9.5 $\mathrm{cm}$ inner diameter and $70 \mathrm{~cm}$ length and the left end is terminated by a insulating plate. The other end of the source tube is attached to a $51 \mathrm{~cm}$ long stainless steel (SS) expansion chamber of $21 \mathrm{~cm}$ inner diameter. The whole chamber is evacuated to a base pressure of $1 \times 10^{-6}$ mbar by a diffusion pump connected to the expansion chamber. The Argon is used as the working gas and experiments are carried out at pressure $1 \times 10^{-3}$ mbar. An $18 \mathrm{~cm}$ long, $\mathrm{m}=+1$ right helicon antenna, placed around the source chamber is used to produce plasma by means of a $13.56 \mathrm{MHz}$ rf power generator through an L-type impedance matching network. The reflected power is kept less than $2 \%$ for all the experiments. The axial location of the antenna center is defined as $\mathrm{z}=0$ and all other axial locations are with reference to this antenna center as shown in Fig. 1]

Five water-cooled electromagnet coils, as shown in Fig. 1, are used to generate an axial magnetic field $\left(B_{0}\right)$. Four different magnetic field configurations are used in our experiments, produced by passing direct current (DC) through different electromagnet combinations. Fig. 22 a shows the axial variation of the simulated magnetic field when $1 \mathrm{~A}$ DC current is passed through different electromagnet combinations to produce four different magnetic field configurations. Fig. $2 \mathrm{~b}$ shows the axial variation of normalized magnetic field with respect to field value at antenna center. Fig. 20 noticeably indicates the magnetic field homogeneity or inhomogeneity in each configurations. The leftmost coil as shown in the Fig. 11 is the first coil and the rightmost coil is the fifth coil. Case A corresponds to a magnetic field profile when $1 \mathrm{~A}$ current is passed through all 5 coils (coil 1-5). The Case $\mathrm{B}$ magnetic field is produced by taking out the first coil and keeping the other 4 (coil 2-5). The Case $\mathrm{C}$ magnetic field is obtained by removing the first and second coils from left and keeping the other 3 coils (coil 3-5). Case D magnetic field corresponds to 1 A current in the fourth and last coil by removing the first three coils from the left.

Two separate single rf compensated Langmuir probes 21 , one straight, inserted from the 


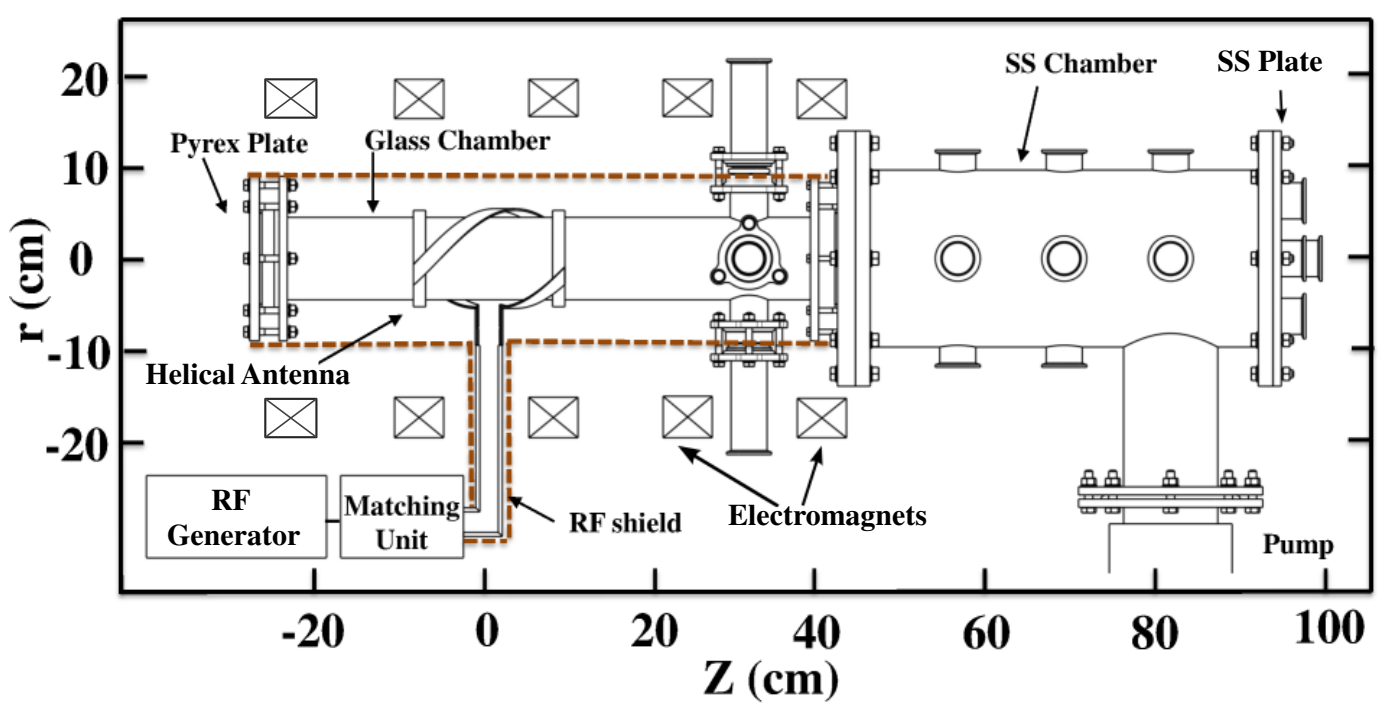

FIG. 1. Schematic diagram of the Helicon plasma experimental setup

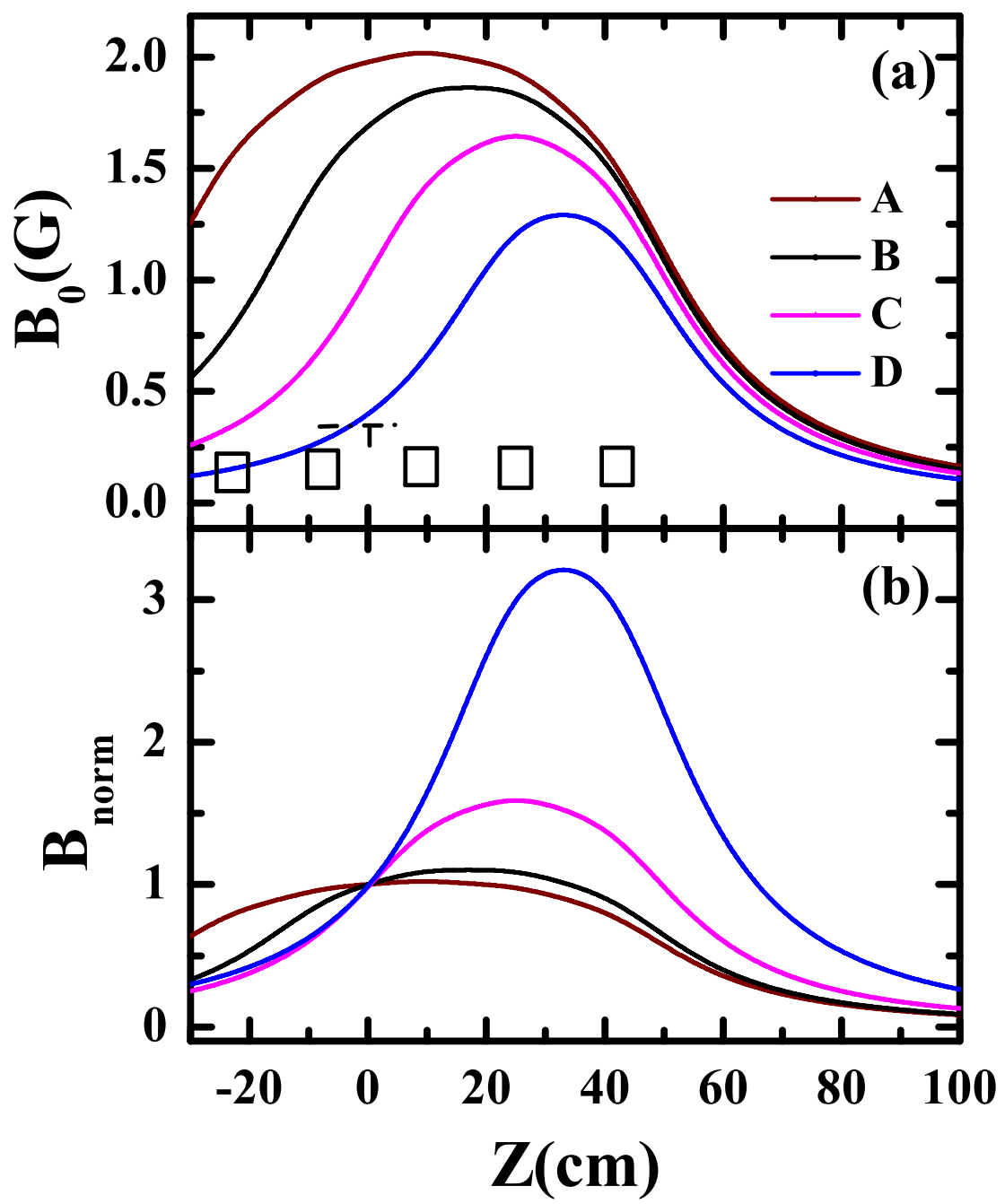

FIG. 2. Axial variation of (a) magnetic field strength and (b) Normalized magnetic field with respect to field value at antenna center for Cases of A-D when $1 \mathrm{~A}$ current is passed in the coils 
top radial port in the source chamber at $\mathrm{z}=31 \mathrm{~cm}$ and other $\mathrm{L}$ shaped, inserted off-axially from the end flange of the expansion chamber at $\mathrm{z}=50 \mathrm{~cm}$, are used to measure the plasma density, $n_{0}$. These locations also correspond to before $\left(z_{\text {before }}=31 \mathrm{~cm}\right)$ and after $\left(z_{\text {after }}\right.$ $=50 \mathrm{~cm})$ both in the magnetic and geometric expansion Fig. 1. The probe tips of nearly equal collection area, are made up of cylindrical tungsten wire of $1 \mathrm{~mm}$ diameter and $4 \mathrm{~mm}$ length. The plasma density determined from the ion saturation current by considering the sheath expansion 22 .

The wave detection is performed by measuring the amplitude and phase of axial wave magnetic field $\left(B_{z}\right)$, by positioning the high frequency B-dot probe about $10 \mathrm{~cm}$ downstream from the antenna center and pulling it back in $2 \mathrm{~cm}$ increments. The B-dot probe is a single loop of $6 \mathrm{~mm}$ diameter made from the shielded coaxial cable of $1.8 \mathrm{~mm}$ outer diameter. At each position, the amplitude is measured as the peak-to-peak value averaged over ten shots. To measure the axial phase variation, the antenna current measured simultaneously using a high frequency Rogowski coil, acts as a phase reference. Both signals (antenna current and B-dot probe) are fed to separate channels of a digital oscilloscope.

\section{EXPERIMENTAL RESULTS AND DISCUSSION}

It is well know that the helicon discharges are quite versatile and depending on operating parameters, the discharge can be operated in capacitive (E), inductive $(\mathrm{H})$ or Helicon wave (W) mode $1,9,23$. To determine the different discharge mode in our experiment, we measured the center plasma density in both source chamber at $\mathrm{z}=31 \mathrm{~cm}$ (Fig. 3 $\mathrm{k}$ ) and expansion chambers at $\mathrm{z}=50 \mathrm{~cm}$ (Fig. 3 $\mathrm{b}$ ). The $n_{0}$ is measured in quasi-uniform magnetic field configuration, i.e. Case $\mathrm{B}$ as function of $\mathrm{rf}$ input power at $1 \times 10^{-3} \mathrm{mbar}$ pressure and B0 $\sim 160 \mathrm{G}\left(I_{B}=87 \mathrm{~A}\right)$ applied magnetic field. Fig. 3 shows the gradual and abrupt changes in center plasma density that can be identified as E-H and H-W transitions near 50 and $700 \mathrm{~W}$ respectively. The discharge mode transitions are also associated with discrete change in values of load capacitor $\left(C_{L}\right)$, Fig. 3 . with optimized reflected power less than $2 \%$ by keeping the fixed value of tune capacitor $\left(C_{T}\right)$ in matching network ${ }^{20}$. The measured values of rms antenna current $\left(I_{a n t}\right)$ as function of rf power is shown Fig. $3 \mathrm{~d}$, it also reflects the mode transitions near 50 and $700 \mathrm{~W}$. It is found that discharge mode transition from $\mathrm{H}$ to $\mathrm{W}$ is clearly observed in source and expansion plasma density for power $700 \mathrm{~W}$. The density jump at $\mathrm{B} 0 \sim 160 \mathrm{G}$ is associated with the helicon wave abortion ${ }^{23}$.

In our previous experimental work ${ }^{19}$ it has been shown that the helicon wave is also absorbed even at low magnetic field $(<100 \mathrm{G})$. The multiple density peaks at low magnetic fields has already been observed in our Helicon Experimental device ${ }^{20}$ when the uniform magnetic field is applied near the antenna in the source chamber. In the present experiments, the phenomenon of density peaking at the low magnetic field is explored by changing the axial magnetic field gradient at the antenna location. The $n_{0}$ measurement is performed in the four different axial magnetic field configurations. These different magnetic field configurations are entitled by the Case A, B, C and D as shown in Fig. 2. The $n_{0}$ is obtained using the rf compensated Langmuir probe at two different axial locations, $\mathrm{z}=31$ $\mathrm{cm}$ and $50 \mathrm{~cm}$. For all present Cases $(\mathrm{A}, \mathrm{B}, \mathrm{C}$, and $\mathrm{D})$ the values of the magnetic field at abscissa are taken at antenna center i.e. $\mathrm{z}=0 \mathrm{~cm}$.

Fig. 4a and Fig. 4b show the $n_{0}$ as function of magnetic field for the Case $\mathrm{B}$ for different rf powers at $\mathrm{z}=31 \mathrm{~cm}$ and $\mathrm{z}=50 \mathrm{~cm}$ respectively. As the magnetic field is slowly increased from $0 \mathrm{G}$, the density rises monotonically till $15 \mathrm{G}$ (at $300 \mathrm{~W}$ ) to $25 \mathrm{G}$ (at $600 \mathrm{~W}$ ), before falling sharply to about half of its peak value near 50-60 G in the source chamber, at $\mathrm{z}=$

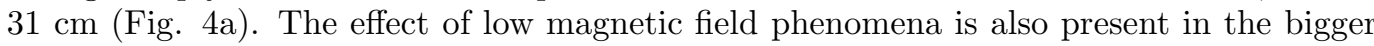
expansion chamber, Fig. 3b. The density rise in the expansion chamber (far away from the antenna) is monotonic till 25-40 G but fall is rather gradual than source chamber. The fall of density with magnetic field is not same in both the chambers may be due different diffusion process involved. At low rf power $(100 \mathrm{~W})$ the very weak density peak is present in both the source and expansion chambers. The magnitude of the peak density is higher and the profile becomes broader for increased rf powers. It is also noted that with increasing 


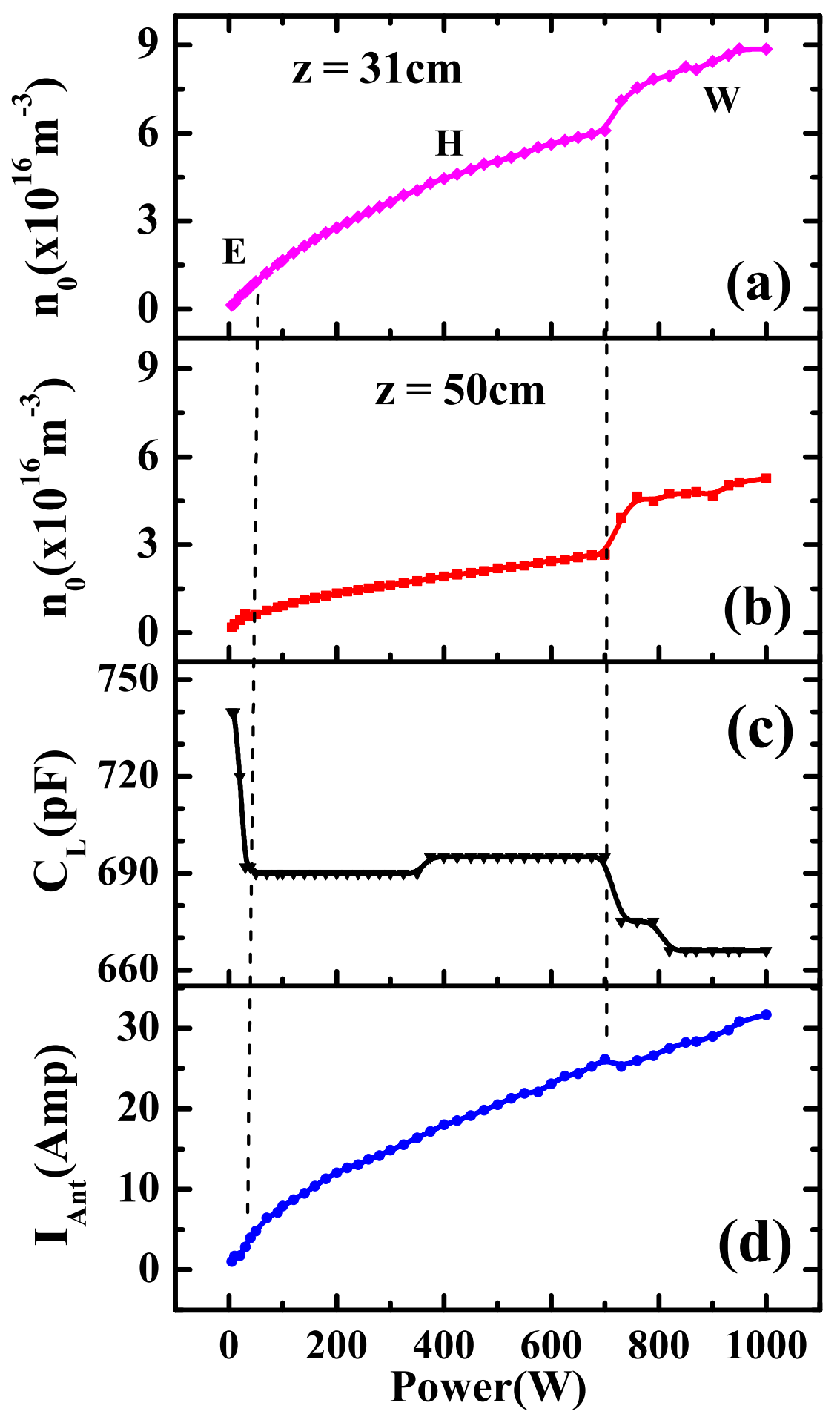

FIG. 3. Helicon discharge mode transitions. Langmuir probe measurement of center plasma density at (a) $\mathrm{z}=31 \mathrm{~cm},(\mathrm{~b}) \mathrm{z}=50 \mathrm{~cm}$ and (c) Value of load capacitor $\left(C_{L}\right)$ and (d) Antenna rms current as function of $\mathrm{rf}$ power, measured at $1 \times 10^{-3} \mathrm{mbar}$ and $B_{0}=160 \mathrm{G}\left(I_{B}=87 \mathrm{~A}\right)$. Dash lines show the capacitive $(\mathrm{E})$ to inductive $(\mathrm{H})$ and inductive to helicon wave $(\mathrm{W})$ mode transitions. 


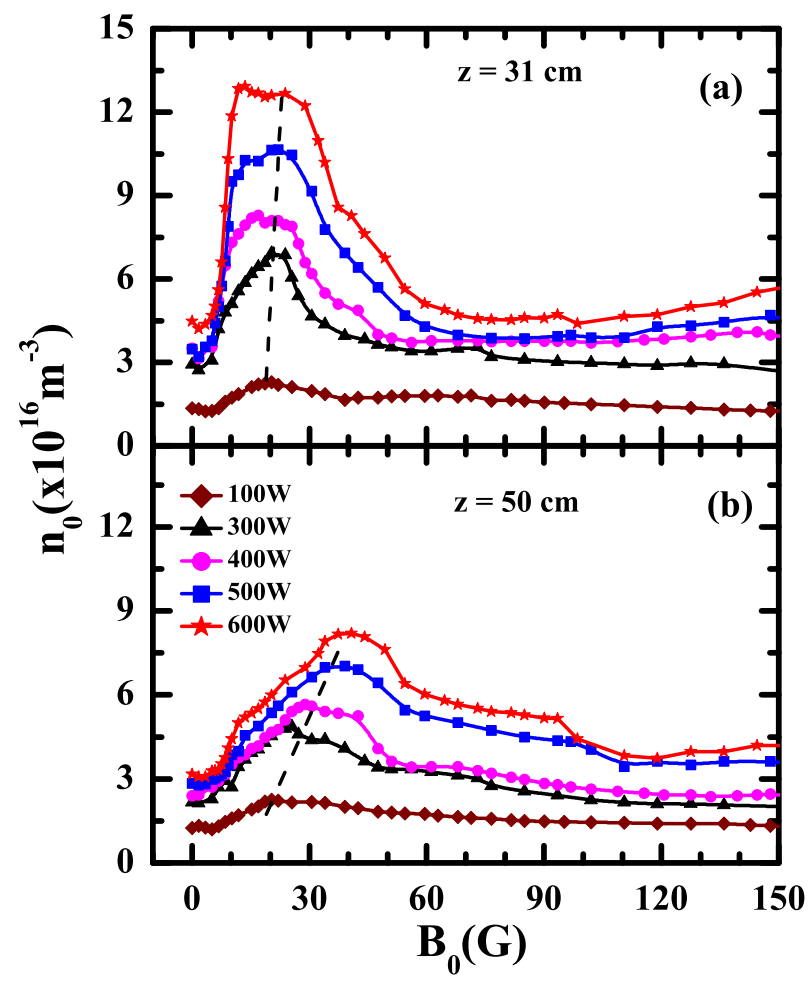

FIG. 4. On-axis variation of plasma density with magnetic field at antenna center for the Case B in the (a) source chamber at $\mathrm{z}=31 \mathrm{~cm}$ and (b) expansion chamber at $\mathrm{z}=50 \mathrm{~cm}$, for different values of rf powers, 100W solid diamonds, 300W solid triangles, 400W solid circle, 500W solid square and 600W solid star.

rf power the density peak shifted to larger magnetic field values. The similar observations can also be found in the previous experiments $15,24,25$.

It has already been established that the density peaking at the low magnetic field is the results of wave coupling rather than capacitive or inductive coupling 17,19. In reference 19 it was shown that the wave propagation near the resonance cone surface causing the density peaking. To establish the existence of helicon wave, the measurement of phase and amplitude of axial wave magnetic field $B_{z}$, are carried out using the B-dot probe. Fig. [5 shows the axial variation of the phase and amplitude of $B_{z}$ at $400 \mathrm{~W}$ rf power in the Case B. At $22 \mathrm{G}$, the axial variation of wave amplitude, Bz (Fig. 5 5 ) has a spatial modulation whereas, phase difference (Fig. 5b) shows the traveling wave character. This type of amplitude modulation can be explained by the beating of different helicon wave modes corresponding to fundamental and higher order radial modes, simultaneously, excited by the antenna11,26,27. These different helicon modes are propagating with different angles of the axial magnetic field. Depending on the magnetic field strength near the antenna, one of these modes can predominantly take part in the ionization process by resonant absorption. An effective traveling wavelength can be found by the slope $\frac{d \phi}{d z}$ of fitted straight line to axial phase variation (Fig. 5b), where $\phi$ is the phase difference. At $22 \mathrm{G}$, in the near field of the antenna the effective traveling wavelength, $\lambda_{\|}=360\left(\frac{d \phi}{d z}\right)^{-1}$, is about $34 \mathrm{~cm}$. However, for the high magnetic field at 50 and $100 \mathrm{G}$, nearly constant phase variation (Fig. 5b) suggested the barely existence of helicon wave. The amplitudes also does not indicate any wave pattern, (Fig. [5 a). This implies that the non-existence of a helicon wave at 50 and $100 \mathrm{G}$ in our experimental conditions in the case B.

The angle of obliquely propagating helicon wave with the axial magnetic field can be found from the relation, $\cos \theta=\frac{k_{\|}}{k}$, where $k=\sqrt{k_{\|}^{2}+k_{\perp}^{2}}, k_{\|}$and $k_{\perp}$ are the total, parallel and perpendicular wavenumbers, respectively. The calculated value of wave propagation 


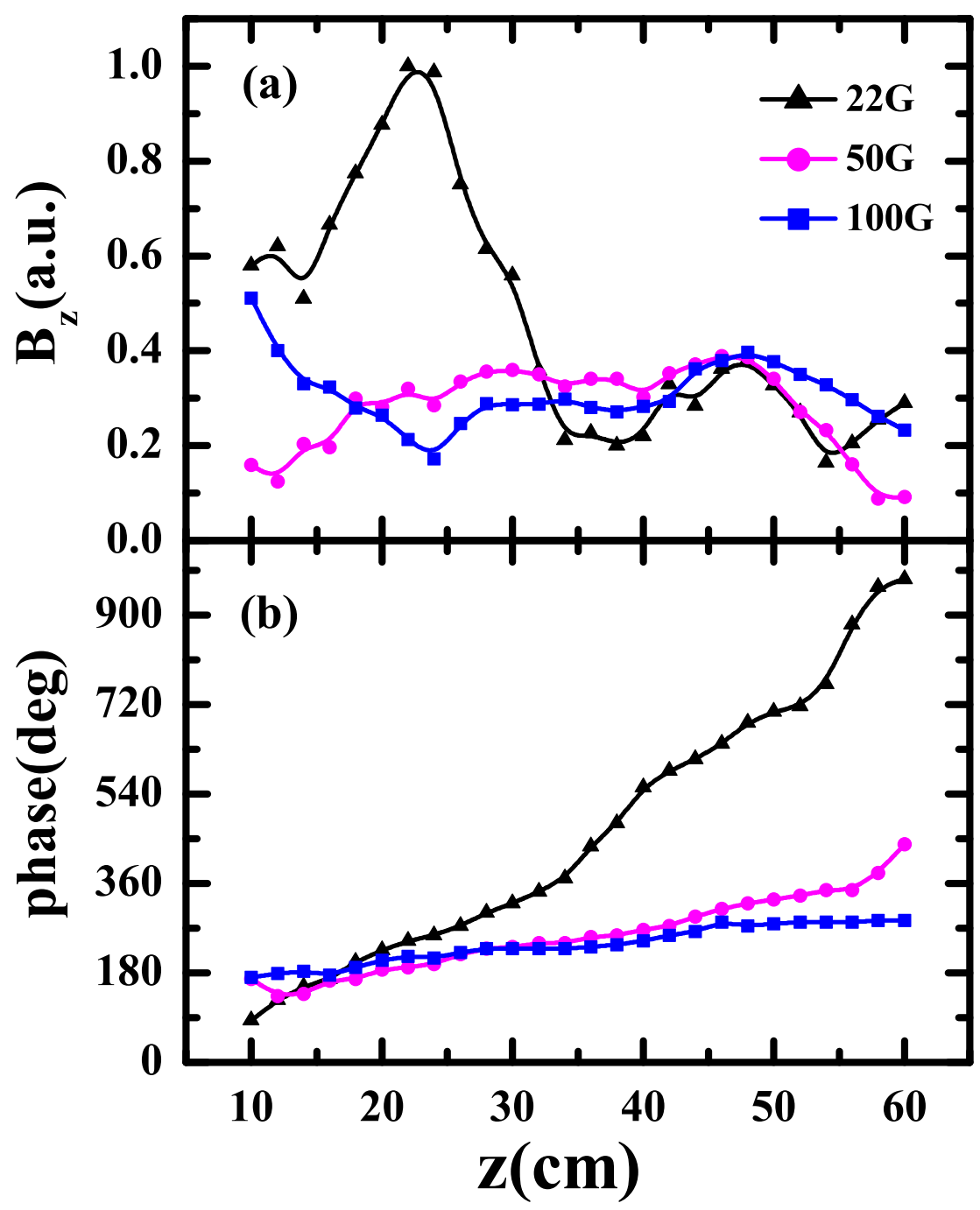

FIG. 5. Axial variation of (a) amplitude and (b) phase of an axial component of wave magnetic field $\left(B_{z}\right)$ at $400 \mathrm{~W}$ rf power and $1 \times 10^{-3}$ mbar for the Case B at magnetic field value $22 \mathrm{G}$, solid triangles, $50 \mathrm{G}$ solid circle and $100 \mathrm{G}$ solid square.

angle is comes about $76.40^{\circ}$ by using the measured values of parallel wavenumber, i.e. $k_{\|}$ $=18.4 \mathrm{~m}^{-1}$ and estimated perpendicular wavenumber $k_{\perp 1}$. The value of $k_{\perp 1}=\frac{3.83}{a}=$ $76.6 \mathrm{~m}^{-1}$ can be found from the $1^{\text {st }}$ root of the first order Bessel function $J_{1}\left(k_{\perp} a\right)$, by considering the plasma radius $a=5 \mathrm{~cm}^{28}$. The calculated value of resonance cone angle $\frac{19}{}$, $\cos \theta=\frac{\omega}{\omega_{c e}}$, for $22 \mathrm{G}$ at which the density peak occurs (Fig. 4 4 ), is comes about $77.30^{\circ}$. It is found that the helicon wave propagation angle well matches with the resonance cone angle. The details of the relation between resonance cone angle and wave propagation angle is described in reference 19 .

The absorption of helicon wave near the resonance cone surface is correlated with the excitation of electrostatic fluctuation ${ }^{29}$. In the present experiment, the measurements of density fluctuation are performed at the magnetic field values where helicon wave present and absent. For this, an electrostatic probe (rf compensation Langmuir probe) biased in the ion saturation region, is placed in the source chamber at $r, z=0,31 \mathrm{~cm}$. A 14-bit PXI based data acquisition system is used to acquire the time series signal with low pass filter of $1.9 \mathrm{MHz}$ with $50 \mathrm{k}$ record length at the sampling rate of $100 \mathrm{kS} / \mathrm{s}$. The frequency spectrum (FFT) of density fluctuation at $400 \mathrm{~W}$ rf power and $1 \times 10^{-3}$ mbar fill pressure for various 


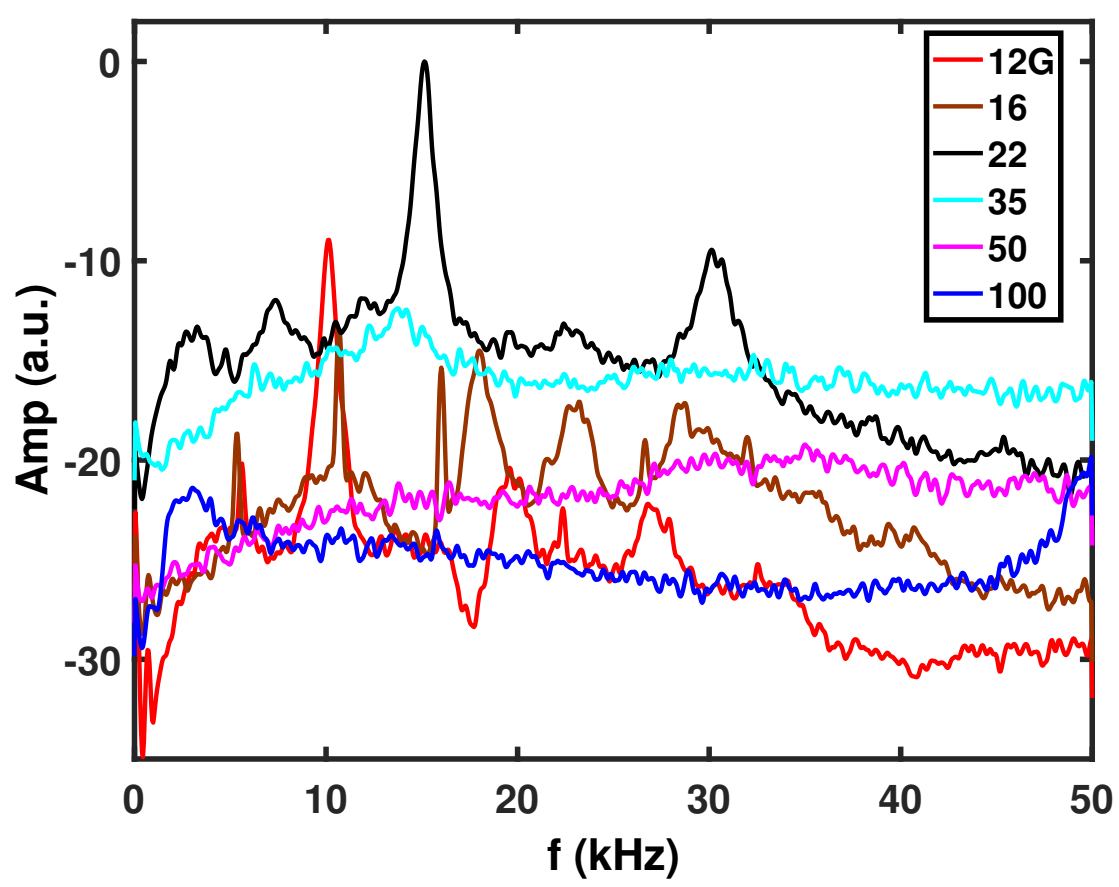

FIG. 6. The frequency spectrum of plasma density fluctuation obtain at the different magnetic field with $\mathrm{rf}$ compensated Langmuir probe in Case B at on-axis location of $\mathrm{z}=31 \mathrm{~cm}, 400 \mathrm{~W} \mathrm{rf}$ power and $1 \times 10^{-3}$ mbar fill pressure.

magnetic field strength in the Case B is shown in Fig. 6] The strong peaks are observed between 10 to $20 \mathrm{kHz}$ frequency at $22 \mathrm{G}$ of magnetic field value, above this there is no significant peak in the frequency spectrum. The presence of electrostatic fluctuations at low magnetic field $(\sim 25 \mathrm{G})$ are associated with the density peaking (Fig. 4) where helicon wave also exist. The magnetic field $>25 \mathrm{G}$, the non-existence electrostatic fluctuations is consistent with non-propagation of helicon wave (Fig. 5) and hence this can be correlated to density fall Fig. 4

Fig. 7 shows the variation of density fluctuation level with an applied magnetic field for two different powers at pressure $1 \times 10^{-3}$ mbar. It is observed that level of fluctuation increases significantly at those magnetic fields where the density peaks occur. Therefore in our experiments, the oblique helicon wave propagation near the resonance cone boundary excites the non-convective electrostatic wave $19,29-31$ which transfers the energy from helicon wave to the plasma creating density peaks at specific magnetic field. This process is responsible for density peaking at the low magnetic field in our experiments.

After establishing the presence of the helicon wave when density peaked at low magnetic field in the quasi-uniform magnetic field configuration, the measurement of plasma density in the source chamber is made for other different magnetic field configuration as shown in Fig. 2 by Case A, B, C, and D. In all the Cases the magnetic field is varied and density is measured at the location, $\mathrm{z}=31 \mathrm{~cm}$ at $1 \times 10^{-3}$ mbar pressure at $600 \mathrm{~W}$ (Fig. 8 $\mathrm{a}$ ) and $300 \mathrm{~W}$ (Fig. 8 8 ). To get the same magnetic field at the antenna center $(\mathrm{z}=0)$, different current is passed for different coil configurations. As the coils are removed, it can be seen from Fig. 2 that field non-uniformity is increases from Case A to Case D. For Cases A, B and C the density is peaked around 17-22 G and the peak value increases as non-uniformity increases for the Case A to C. With removing the one more coil from the case $\mathrm{C}$, the magnetic field near the antenna become more non-uniform or diverging in the Case D. In the case D, the density peaked around 50-60 G and increases about five times than the uniform magnetic field case at the same magnetic field value. Fig. 9 shows the density at 50G, increases as magnetic field non-uniformity increases from case A to D. The plasma production efficiency, ${ }^{32} \frac{N_{e}}{p_{r f}}$ which is the ratio of number of total electrons to input 


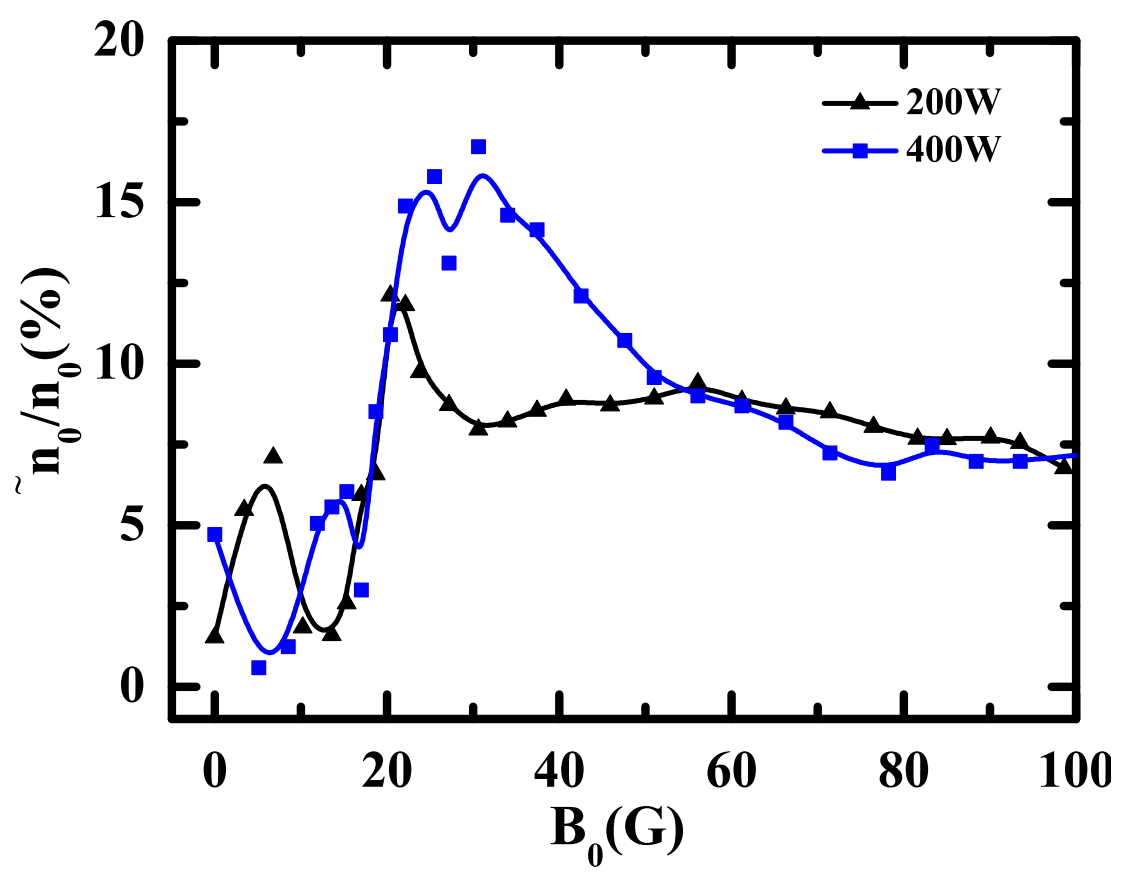

FIG. 7. Amplitude variation of density fluctuation with the external magnetic field as a function of $\mathrm{rf}$ powers in Case $\mathrm{B}$ at the location of $r, z=0,31 \mathrm{~cm}$ and $1 \times 10^{-3}$ mbar fill pressure. $200 \mathrm{~W}$ solid triangles and $400 \mathrm{~W}$ solid square.

power is also increases with the magnetic field inhomogeneity. Fig. 10 shows the production efficiency estimated at on-axis at $\mathrm{z}=31 \mathrm{~cm}$ in all four cases. To estimate the production efficiency, the production region is important, i.e. plasma core instead of plasma edge. It is seen from above discussion that the inhomogeneous magnetic field near the antenna location improves the plasma production significantly.

Our results show a systematic variation of plasma density with magnetic field inhomogeneity. Plasma production is more when the magnetic field is more diverging near the antenna. This kind of behavior of increase of production efficiency with magnetic field divergence may be attributed to multimode oblique cyclotron resonance. In the direction of the magnetic field, the $\mathrm{m}=+1$ right helicon antenna is supposed to excite different axial and radial eigenmodes 27,33 . Depending upon the magnetic field near the antenna all these different modes can simultaneously couple to different helicon modes and couple power in the plasma. In our earlier experiments 19 it was shown that multiple modes can be excited at different uniform magnetic fields near the antenna. These modes can have oblique cyclotron resonance for a critical magnetic field which satisfies $\cos \theta=\frac{\omega}{\omega_{c e}}=\frac{k_{\|}}{k}=\frac{k_{\|}}{\sqrt{k_{\|}^{2}+k_{\perp}^{2}}}$. If multiple modes are present, they will have multiple resonances at different critical magnetic fields. With a uniform magnetic field, only a single resonance is possible for a mode. When a non-uniform magnetic field (because of the divergence) is applied, different magnetic field values are available near the antenna near field for different eigenmodes resonance conditions. Among all the possible excited modes some modes can have resonances. This will lead to more power absorption and hence rise in the efficiency of the source as observed in the present experiment. Another interesting feature to be noted here is that the width of the peaks of density increases as the non-uniformity in the magnetic field is increased. This is indicative of the presence of different resonating modes. As low field helicon sources are proposed recently for space propulsion ${ }^{5.6}$, using a highly diverging magnetic field near the antenna may be better suited in terms of efficiency of the source. It will reduce the weight of the thrusters as well as the power consumption.

Fig. 11 shows the on-axis plasma density for the Case B and D at $400 \mathrm{~W}$ rf power. The plasma density is peaked around 40-50 G in the Case D and it is nearly 6 times higher 


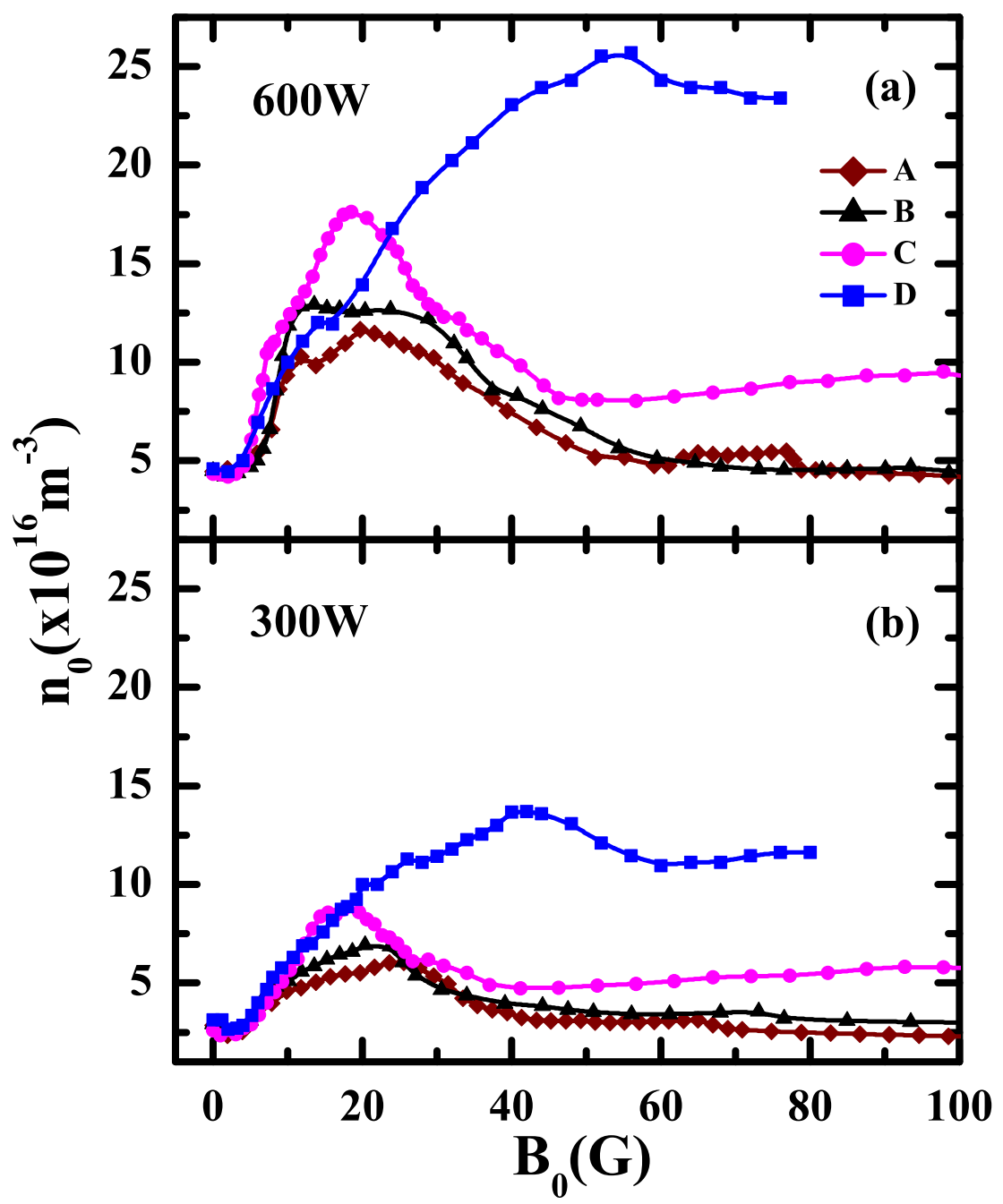

FIG. 8. Variation of plasma density in the source chamber at $\mathrm{z}=31 \mathrm{~cm}$ with the applied magnetic field at antenna center for Cases A-D; at pressure $1 \times 10^{-3}$ mbar and rf powers (a) 600W and (b) $300 \mathrm{~W}$.

than the Case B at that magnetic field value. To investigate the presence of multimode in the inhomogeneous magnetic field configuration, the axial profiles of the wave amplitude and phase of $B_{z}$ are measured in the Case $\mathrm{D}$ and comparisons are made with the quasiuniform magnetic field configuration, i.e. Case B. Fig. 11] shows the density variation with magnetic field for Case $\mathrm{B}$ and $\mathrm{D}$ at which wave amplitude and phase of $B_{z}$ are measured. Fig. 12 shows the axial variation of the amplitude (Fig. 12 and c) and the phase difference (Fig. 12 $\mathrm{b}$ and d) of $B_{z}$ at two different magnetic field values, $22 \mathrm{G}\left(\frac{\omega_{c e}}{\omega_{r f}}=4.5\right)$ and 50 $\mathrm{G}\left(\frac{\omega_{c e}}{\omega_{r f}}=10\right)$, respectively for the Case B (solid triangle) and D (solid square) at $400 \mathrm{~W}$ rf power and $1 \times 10^{-3}$ mbar fill in pressure. At $22 \mathrm{G}$, for both cases $\mathrm{B}$ and $\mathrm{D}$, the phase difference variations (Fig. 12a) show the traveling wave character and amplitude (Fig. [12b) modulations show the beating wave pattern. The beating pattern $11,26,27$ of amplitude in presence of propagating wave indicates the existence of more than one (radial or axial) mode in our experiment. At $50 \mathrm{G}$, for Case B (where density has 5 times lower magnitude from the Case D, Fig. 11), the phase difference has no significant axial variation (Fig. 12 $\mathrm{d}$, solid triangles) i.e. wave propagation may ceases at this magnetic field value. The contrasting results are observed in the Case D at $50 \mathrm{G}$ (where the density is peaked), where $B_{z}$ has amplitude modulation (Fig. 12k, solid square) and the phase oscillates (Fig. 12 $\mathrm{d}$, solid 


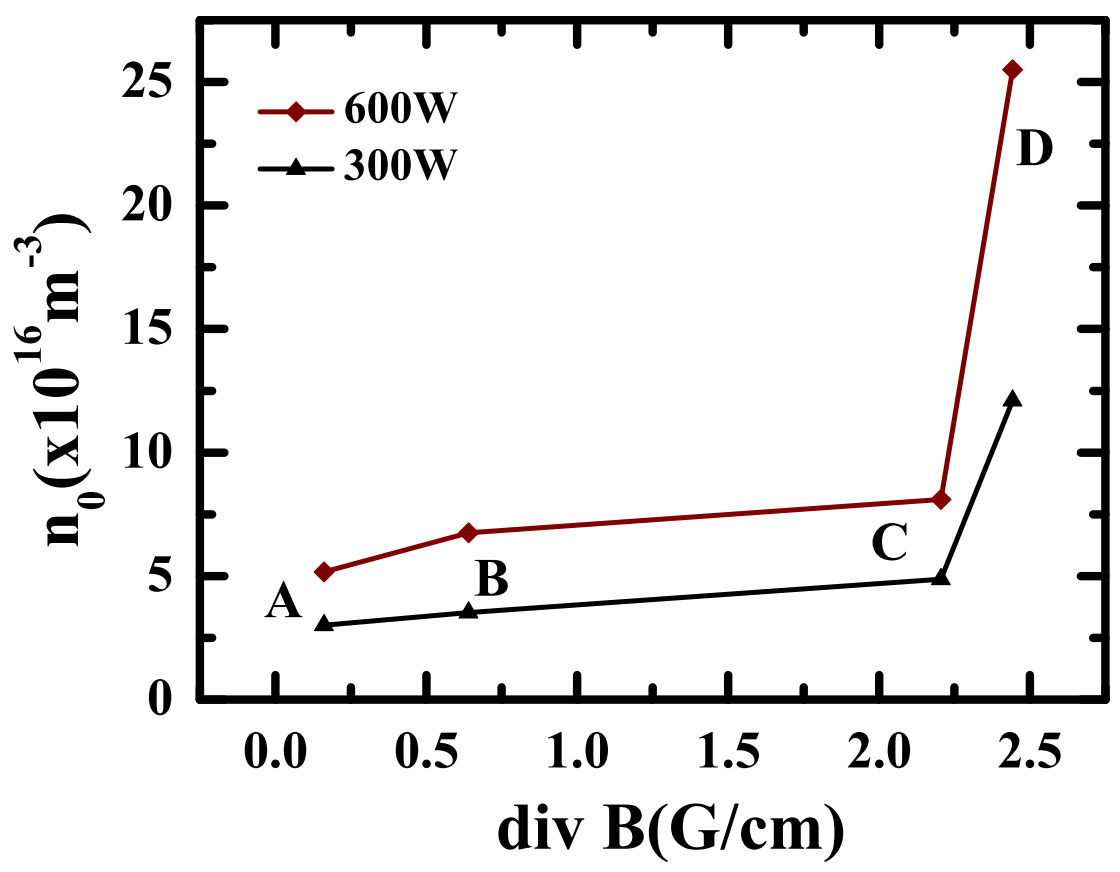

FIG. 9. Density as function of magnetic field non-uniformity in different Cases at $50 \mathrm{G}$ and $1 \times 10^{-3}$ mbar pressure.

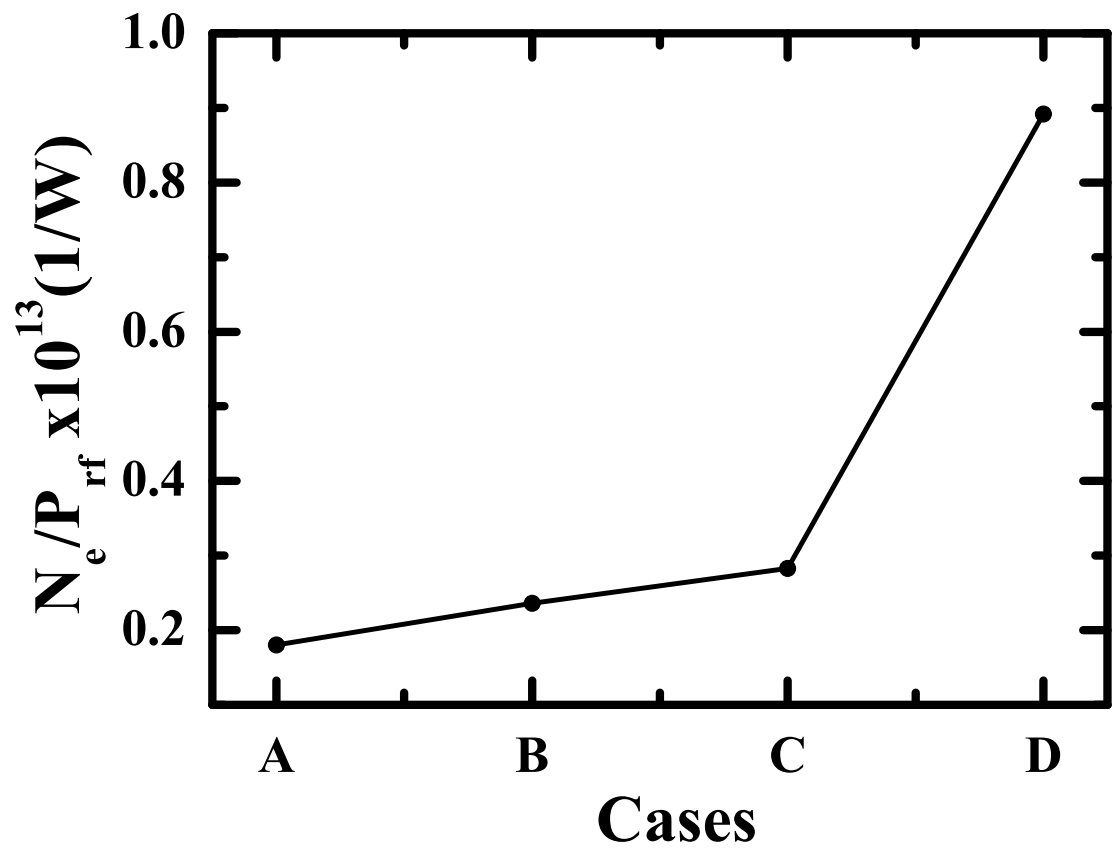

FIG. 10. On-axis plasma production efficiency at $\mathrm{z}=31 \mathrm{~cm}$, in different Cases at 50G, 600W and $1 \times 10^{-3}$ mbar pressure.

square) substantially with the position. It seems that, in the Case D, helicon wave exist along the entire axial distance including the expansion chamber and has partially traveling and partially standing wave structure. This implies that waves can have the interference of multiple radial or axial modes. This results in a standing wave field structure ${ }^{27,34,35}$ that gives rise to the nearly constant phase signal at $25<\mathrm{z}<45 \mathrm{~cm}$ in the Case D, Fig. 12 $\mathrm{d}$. 


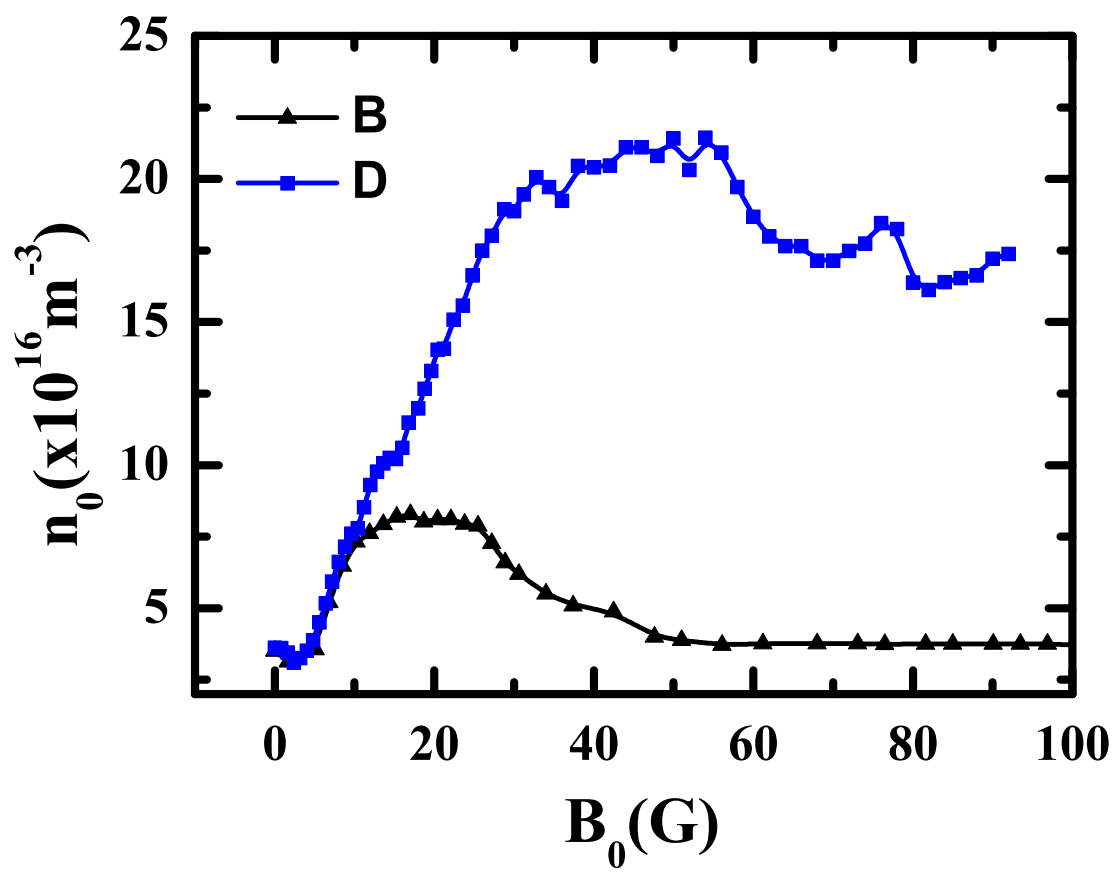

FIG. 11. On-axis variation of plasma density with the magnetic field at $400 \mathrm{~W}$ RF power and $1 \times 10^{-3}$ mbar fill pressure. Case B (solid triangle) and Case D (solid square).
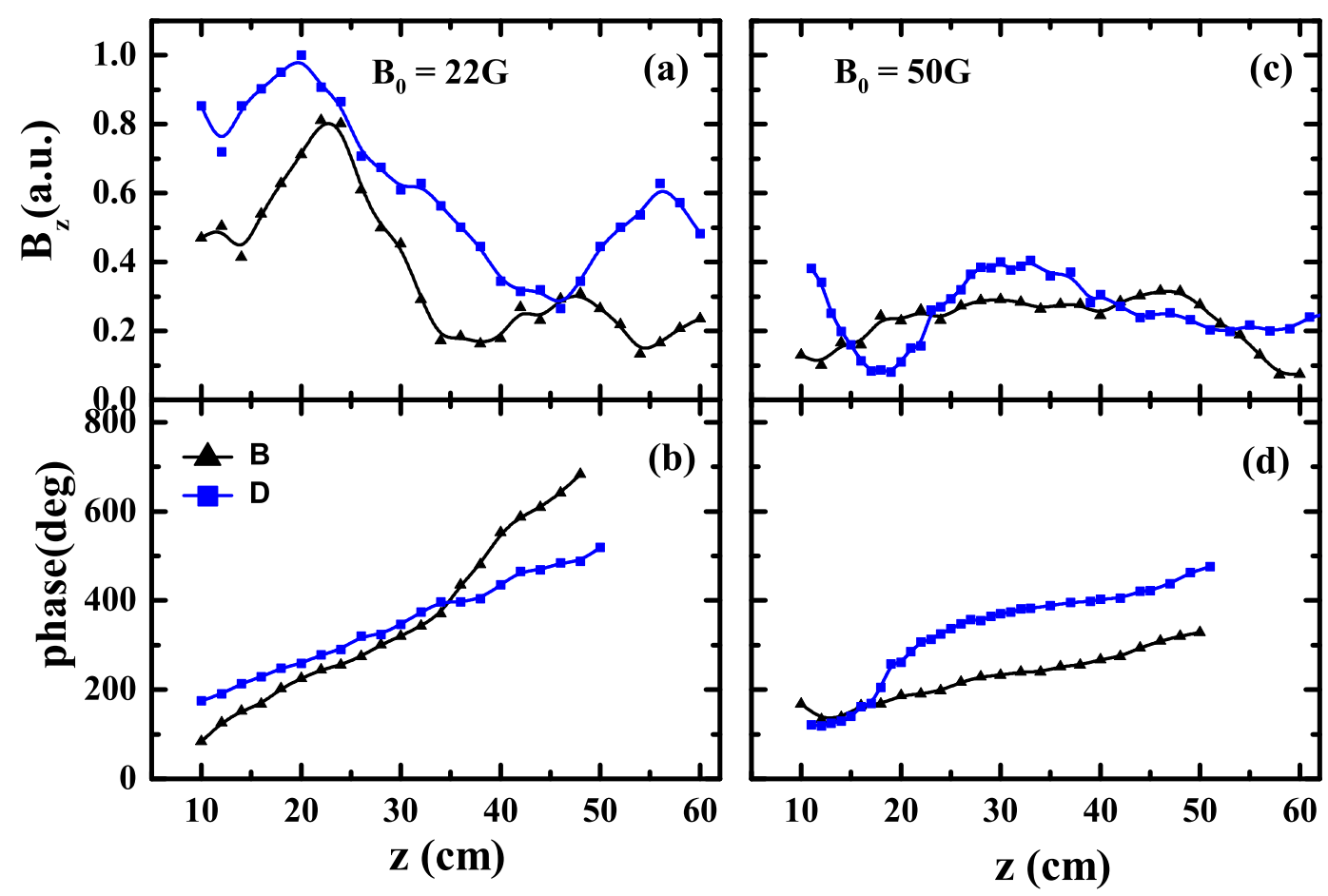

FIG. 12. Axial variation of amplitude and phase of $B_{z}$ for Case B and D magnetic field configuration for $22 \mathrm{G}$ (a and b) and $50 \mathrm{G}\left(\mathrm{c}\right.$ and $\mathrm{d}$ ) at $400 \mathrm{~W}$ rf power and $1 \times 10^{-3}$ mbar fill pressure. 


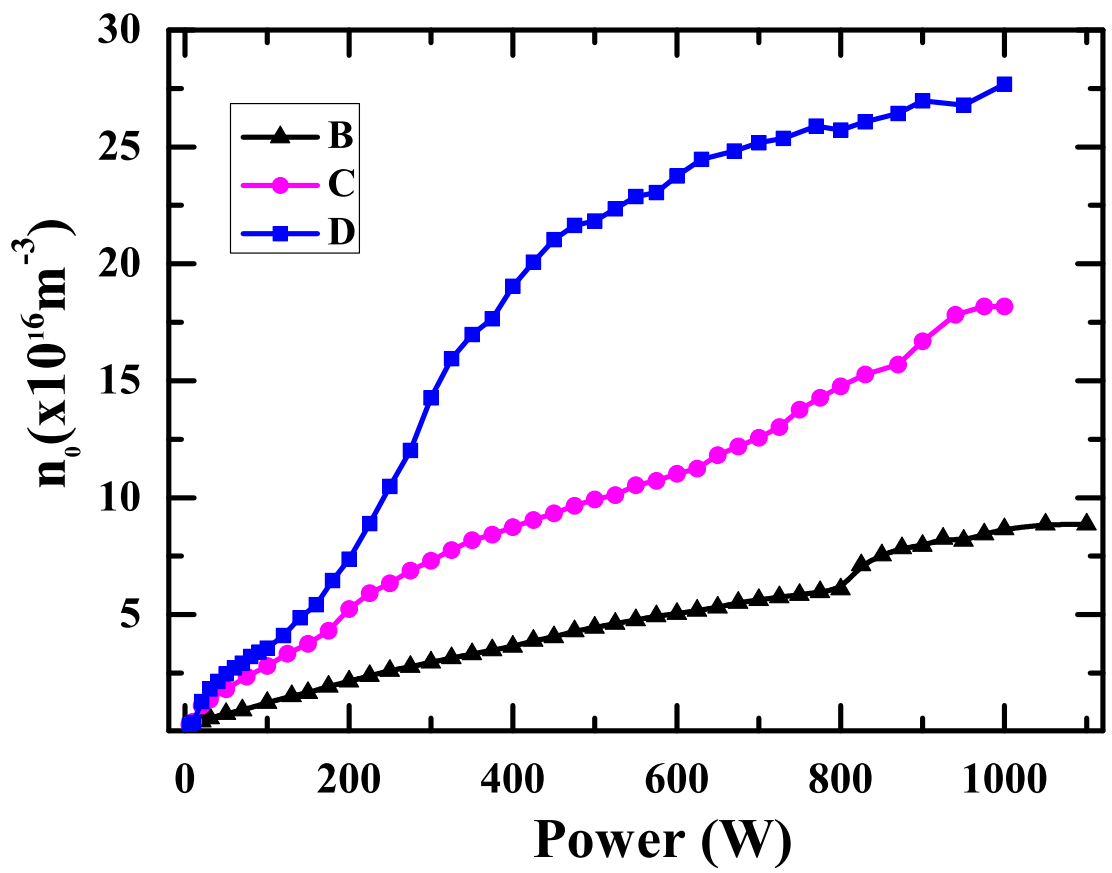

FIG. 13. Variation of on-axis plasma density at $\mathrm{z}=31 \mathrm{~cm}$ with $87 \mathrm{~A}$ DC current for the Cases of $\mathrm{B}$ (solid triangle), $\mathrm{C}$ (solid circle) and D (solid square) at pressure $1 \times 10^{-3}$ mbar.

The multiple modes exist due to presence of different exciting condition corresponding to availability of broad range of magnetic field near the antenna, Case D Fig. 2.

Fig. 13 shows the variation of on-axis plasma density with RF powers for the Cases of $\mathrm{B}, \mathrm{C}$, and $\mathrm{D}$ at $1 \times 10^{-3}$ mbar fill pressure. The density in the Cases B, C and D is obtain by keeping the fixed DC current, $87 \mathrm{~A}$, which corresponds to magnetic field values at the antenna center about 150, 90 and 34 G, respectively. It is clear from the Fig. 13 that, above $300 \mathrm{~W}$ rf powers, in the Case $\mathrm{D}$, the density is five times higher than the Case B. The density at $1000 \mathrm{~W}$, in case B is about $8 \times 10^{16} \mathrm{~m}^{-3}$ which can be achievable at $200 \mathrm{~W}$ in the Case D. These results successfully demonstrate that the higher ionization can be achieved by careful placing of electromagnet or permanent magnet near the antenna. This will effectively reduce the coast of the magnetic power supply and power consumption which in turn enhanced helicon discharge efficiency

\section{SUMMARY AND CONCLUSION:}

Four different magnetic field configurations near the helicon antenna are used to look for the effect of magnetic field inhomogeneity. It is shown that plasma production efficiency increases with increasing the magnetic field inhomogeneity. The results are explained on the basis of multimode excitation due to the presence of a wide range of magnetic fields nearby the antenna. The widths of the peaks in density increase with non-uniformity of the applied magnetic field. This is indicative of multimode resonance absorption leading to higher efficiency. As the less coil is more efficient, the cost of the source will be reduced drastically.

${ }^{1}$ P. A. J., V. D., and B. R. W., "The application of the helicon source to plasma processing," Journal of Vacuum Science \& Technology B: Microelectronics and Nanometer Structures Processing, Measurement, and Phenomena 9, 310-317 (1991).

${ }^{2}$ T. Mieno, T. Kamo, D. Hayashi, T. Shoji, and K. Kadota, "Efficient production of o+ and o ions in a helicon wave oxygen discharge," Applied Physics Letters 69, 617-619 (1996).

${ }^{3}$ H. D. Jung, M. J. Park, S. H. Kim, and Y. S. Hwang, "Development of a compact helicon ion source for neutron generators," Review of Scientific Instruments 75, 1878-1880 (2004). 
${ }^{4}$ C. Charles, "Plasmas for spacecraft propulsion," Journal of Physics D: Applied Physics 42, 163001 (2009).

${ }^{5}$ F. F. Chen, "Permanent magnet helicon source for ion propulsion," IEEE Transactions on Plasma Science 36, 2095-2110 (2008).

${ }^{6}$ J. Ling, M. D. West, T. Lafleur, C. Charles, and R. W. Boswell, "Thrust measurements in a low-magnetic field high-density mode in the helicon double layer thruster," Journal of Physics D: Applied Physics 43, 305203 (2010)

${ }^{7}$ T. Harle, S. J. Pottinger, and V. J. Lappas, "Helicon double layer thruster operation in a low magnetic field mode," Plasma Sources Science and Technology 22, 015015 (2013)

${ }^{8}$ S.-B. C, C. C, and Boswell, "Low temperature growth of nanocrystalline tio2 films with ar/o2 low-field helicon plasma," Surface and Coatings Technology 205, 3939 - 3946 (2011).

${ }^{9}$ D. A. W., J. C. O., B. R. W., and E. A. R., "Plasma production from helicon waves," Physics of Plasmas 3, 2788-2796 (1996).

${ }^{10}$ F. F. Chen, X. Jiang, J. D. Evans, G. Tynan, and D. Arnush, "Low-field helicon discharges," Plasma Physics and Controlled Fusion 39, A411 (1997)

${ }^{11}$ L. T., H. N., M. J., S. W., and K. G. H., "Azimuthally symmetric pseudosurface and helicon wave propagation in an inductively coupled plasma at low magnetic field," Physics of Plasmas 5, 3135-3142 (1998).

${ }^{12}$ W. S. J, K. J. G, K. C. B, and K. S. K, "Observation of enhanced negative hydrogen ion production in weakly magnetized rf plasma," Physics Letters A 313, 278 - 283 (2003).

${ }^{13}$ G. Sato, W. Oohara, and R. Hatakeyama, "Experimental characterization of a density peak at low magnetic fields in a helicon plasma source," Plasma Sources Science and Technology 16, 734 (2007)

${ }^{14}$ S. J. Wang, J.-G. Kwak, S. K. Kim, and S. Cho., "Experimental study on the density peaking of an rf plasma at a low magnetic field," J. Korean Phy. Soc. 51, 989-992 (2007).

${ }^{15}$ S. G., O. W., and H. R., "Efficient plasma source providing pronounced density peaks in the range of very low magnetic fields," Applied Physics Letters 85, 4007-4009 (2004).

${ }^{16}$ E. G. S., K. Junghee, and W. Choe, "Wave mode conversion and mode transition in very high radio frequency helicon plasma," Physics of Plasmas 13, 073505 (2006).

${ }^{17}$ T. Lafleur, C. Charles, and R. W. Boswell, "Characterization of a helicon plasma source in low diverging magnetic fields," Journal of Physics D: Applied Physics 44, 055202 (2011)

${ }^{18}$ C. F. F and B. R. W, "Characterization of a helicon plasma source in low diverging magnetic fields," IEEE Trans. Plasma Sci. 25, 1245-1257 (1997).

${ }^{19}$ K. K. Barada, P. K. Chattopadhyay, J. Ghosh, S. Kumar, and Y. C. Saxena, "Observation of low magnetic field density peaks in helicon plasma," Physics of Plasmas 20, 042119 (2013)

${ }^{20}$ K. K. Barada, P. K. Chattopadhyay, J. Ghosh, S. Kumar, and Y. C. Saxena, "A linear helicon plasma device with controllable magnetic field gradient," Rev. Sci. Instrum 89, 063501 (2012).

${ }^{21}$ S. Yadav, S. Ghosh, S. Bose, K. K. Barada, R. Pal, and P. K. Chattopadhyay, "Role of ion magnetization in formation of radial density profile in magnetically expanding plasma produced by helicon antenna," Physics of Plasmas 25, 043518 (2018)

${ }^{22}$ H. I, Principles of Plasma Diagnostics (second edition (Cambridge University Press, 2005)) p. 65 and 67.

${ }^{23}$ C. K. Kyeong, T. E. Sheridan, and R. W. Boswell, "Resonant cavity modes of a bounded helicon discharge," Plasma Sources Sci. Technol. 8, 421-431 (1999).

${ }^{24}$ L. T., C. C., and B. R. W., "Plasma control by modification of helicon wave propagation in low magnetic fields," Physics of Plasmas 17, 073508 (2010)

${ }^{25}$ W. Y., Z. G., L. Z. W., O. J. T., and C. Q., "Two density peaks in low magnetic field helicon plasma," Physics of Plasmas 22, 093507 (2015).

${ }^{26}$ F. F. Chen, I. D. Sudit, and M. Light, "Downstream physics of the helicon discharge," Plasma Sources Science and Technology 5, 173 (1996).

${ }^{27}$ L. Max, S. I. D., C. F. F., and A. Donald, "Axial propagation of helicon waves," Physics of Plasmas 2, 4094-4103 (1995)

28

${ }^{29}$ R. Boswell, "Non-convective parametric instability associated with whistler wave resonance cone,"

${ }^{30}$ M. Krmer, Y. M. Aliev, A. B. Altukhov, A. D. Gurchenko, E. Z. Gusakov, and K. Niemi, "Anomalous helicon wave absorption and parametric excitation of electrostatic fluctuations in a helicon-produced plasma," Plasma Physics and Controlled Fusion 49, A167 (2007)

${ }^{31}$ A. Akhiezer, V. Mikhailenko, and K. Stepanov, "Ion-sound parametric turbulence and anomalous electron heating with application to helicon plasma sources," Physics Letters A 245, 117 - 122 (1998).

${ }^{32}$ S. Shunjiro and T. Takao, "Development of very large helicon plasma source," Review of Scientific Instruments 75, 1941-1946 (2004).

${ }^{33}$ K. I. V. and B. G. G., "An evaluation of different antenna designs for helicon wave excitation in a cylindrical plasma source," Physics of Plasmas 3, 4396-4409 (1996).

${ }^{34}$ T. S. M., D. C. Mark, S. J. E., and A. Kamran, "Optical, wave measurements, and modeling of helicon plasmas for a wide range of magnetic fields," Physics of Plasmas 11, 878-887 (2004)

${ }^{35} \mathrm{~K}$. Takahashi, A. Chiba, and A. Ando, "Modifications of wave and plasma structures by a mechanical aperture in a helicon plasma thruster," Plasma Sources Science and Technology 23, 064005 (2014) 